\title{
VMS Software for the Jorway-411 Interface
}

T. Dorries, C. Moore, R. Pordes, and V. White

Data Acquisition Software Group

Fermi National Accelerator Laboratory

P.O. Box 500, Batavia, Illinois 60510

May 1987

*Presented at the Fifth IEEE Conference on Real-Time Computer Applications in Nuclear, Particle, and Plasma Physics, San Francisco, California, May 12-14, 1987. 
Terry Dorries, Carmenits Moore Ruth Pordes, Vicky White

Data Acquisition Software Group?

Fermi National Accelerator Laboratory*

P.O. Box 500, Batavia, Il 60510

Introduction

This reports on a Software Package to access CAMAC through the Jorway-411 hiterface, for use on $\mathrm{VAX} / \mathrm{VMS}$ systems. The software can be used to acress parallel and/or seria) CAMAC branch highways, and multiple Jorways may be connected to the VAX UNIBUS or MicroVax QBUS. The software available includes a VAX/VMS device driver for the JORWAY-4II' and support routines and programs that arcess the driver. The software is accompanied by extensive documentation.

As discussed below, several of the packages originated from other institutions and have been enhanced by the Data Acquisition Software Group at Fermilab. The software package is in widespread use at Fermilab and over 20 other sites.

The software can be run on any of the UNIBUS VAX-11 family of computers using a UNIBUS Jorway-411 to interface to CAMAC, or on a Microvax-II either interfaced to CAMAC through a Q-BUS Jorway-411, or through a Microverter and UNIBUS Jorway. The package includes facilities for performing CAMAC operations remotely. The $V A X$ on which the user runs his or her program need not be directly connected to the Jorway. Server programs allow a VAX connerted via DECNET or a PDP-11 connected via a DR1l-W link ${ }^{2}$ to be the route for accessing the CAMAC hardware.

The CAMAC VMS software consists of the components as shown in Fig. 1.

\section{CAMAC Device Driver}

A VAX/VMS Device Drjver, CJDRIVER, provides multi-user access to a Jorway, as well as provision for data acquisition from CAMAC - where a CAMAC LAM stimulates execution of a list of CAMAC operations. The driver incorporates the original SLAC CAMAC driver for the Jorway by Charles Granieri ${ }^{3}$. It provides for single word and DMA operations to be performed. added:

At Fermilab the following features have been

\section{A Module and Crate Booking Handler.}

A user may request exclusive access to one or more partictiar CAMAC Crates or stations within a crate. The CAMAC driver keeps lists of the crates and stations booked by each process and channel identifies. When the booking handler is instalied. any CAMAC operation attempted through the normal CAMAC driver is only done if the process is allowed access to the crate and station. A very simple priority and identification scheme aliows for restricted as weli as exclusive access to a booked station, and for priarity override of a booked module ${ }^{4}$.

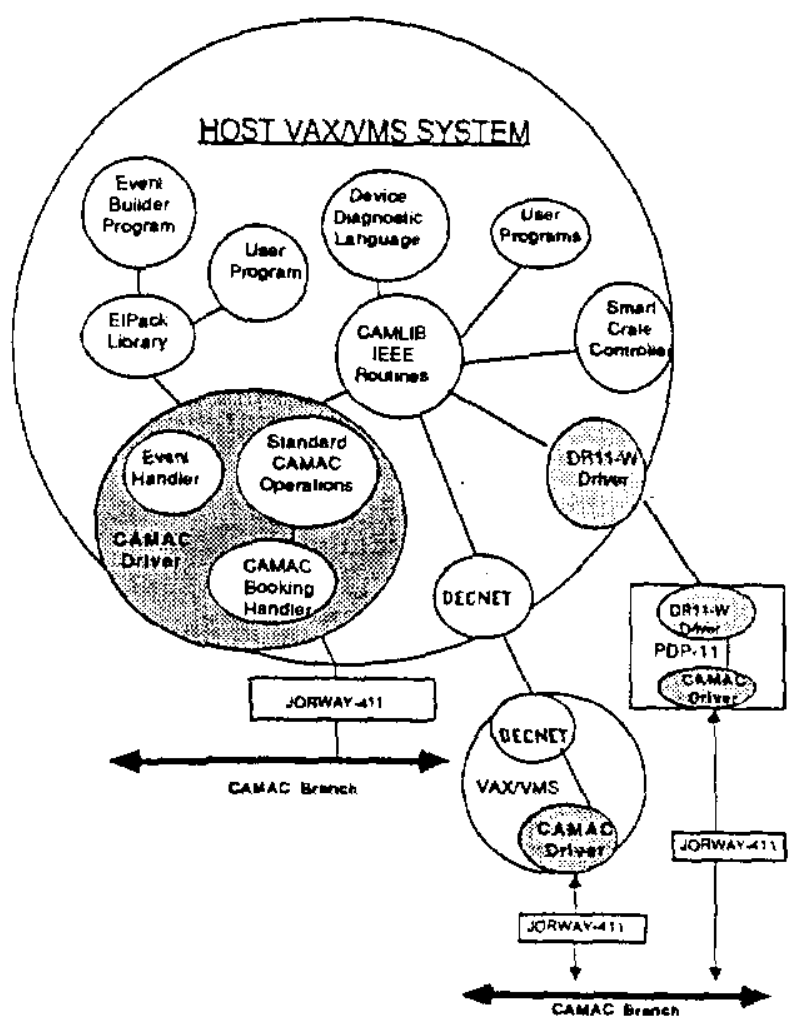

Figure 1: Overview of the CAMAC-VMS Software Product

\section{Event Handler Module}

A whole parallel piece of code has been added to the driver to provide data acquisition functions.

The Event Handler allows a program to be informed of the occurrence of a CAMAC LAM. In addition, the user may pre-dechare lists of CAMAC operations to be performed on receipt of a specific CAMAC IAM or on expiration of a softwarc timer. The user aiso provides a data buffer into which data read by these lists will be read. The driver stores the address of a connected list. The user then declare a data buffer to be filled by one or more executions of the list. When the CAMAC driver detects tine LAM. it executes the CAMAC list and reads datia into the bufier. When sufficient number of events have been read to fill the buffer. the user is informed and can access the event data.

This mechanism significantly reduces the VMs QIO and softwate overhead associated with the

"Operated by the TIA. Inc., under contract with the U.S. DOE. 
CAMAC readout. Only 2 QIOs are sequired to read out as many events as can fin in the declared buffer and to execute any number of CAMAC operations. Hecalse of the specialized purpose of the Event Hajdler package, the lists are restricied to no-data CAMAC operations, CAMAC write operations where the data can be specified when the list is built. and read operations. No arithmetic, logical or other general programming functions are supported by the list structurc.

The Event Handler allows multiple LAMs to be actively connected to different processes at any times.

\section{Support Routine Packages}

Easy user access to each part of the CAMAC driver is provided by Fortran subroutine packages.

\section{IEEE Standard Routine Package}

An implenentation of the IEEE CAMAC routines ${ }^{6}$ provides access to the original SLAC driver. All flavours of CAMAC DMA are implemented $Q$ STOP, Q-REPEAT and Q-SCAN. This library, CAMLIB, is an extension of that implemented by the University of Michigan. ${ }^{12}$

Extensions to the original package and to the IEEE specification, allow access to more than one CAMAC branch, and access to both parallel and serial branch highways, access to the Booking Handicr, and extensive error reporting ${ }^{7}$. Further extensions provide a transparent interface to the remote CAMAC facility described below, and to the Fermilab Smart Crate Controller, described in a companion paper to this conference. The changing of a VAX/VMS logical, external to the user's program, is all that is needed to switch between the various CAMAC interfaces.

\section{Event Handier Packages}

A package of Fortran subroutines allows a user program to dynamically construct and declare the CAMAC lists. A program which wes the subroutines has been written to aliow the user to construct the CAMAC lists as files of ASCll iext and pass them through an interpreter before acciaring them to the drivers.

\section{Remote CAMAC Functions}

A program running on a VAX/VMS system may actually execute the CAMAC operations on a CAMAC branch physically connected to another $V A X$, connected to the user's VAX through DECNET or on a PDP-i1 running RSX-11M and connected by a DRII-W ink. Tise PDP-I1 and $V A X$ must be running the Fermilab Connected Machines software package. Server programs are provided to run on the remote VAX or $R S X-11 M$ system. Although the performance of this configuration is much slower than that for CAMAC directly connected to the users VAX, this facility has been found to be useful in installations where experiments are taking data on one or more PDP-1ls. and where calibration and monitoring programs are more naturalyy suited for a host l'AX'?

\section{Auplication Level Programe}

The software is accompanied by sample programs which exercise each feature of the CAMAC: driver. The interartive, interprelive program Device Diagnostic Language ${ }^{10}$ has been interfaced to the IEEE CAMAC routines. The Formilab $Y A X$ data acquisition program. EVENT BlildDER, has becu interfaced to allow CAMAC data to be taken and concatenated with event data from other sources ${ }^{i n}$

\section{Timings}

The timings measured depend on which VAX system is being used. On a VAX 11/780 CAMAC DMAs take between 3.5 and 5 microseconds per 16-bit word. On \& Microvax II system (using either a QBus Jorway-411 or a UNIBUS Jorway interfuced through a Microverter) DMA specds of 2.5 microseconds per 16 bit word have been measured.

The Q1O overhead for a normal CAMAC opcration is $5 \mathrm{msec}$ on a MicroVax-II and $6 \mathrm{msec}$ on a VAX-11/780. The event handler overhead is about 10 usec per list operation. The QIO overhead for event readout depends significantly on the data buffer size.

\section{Summary}

Traditionally, Fermilab fixed target experiments have read their CAMAC data into PDP-1. computers. They have used the VAX software te perform calibration and monitoring tasks on the experiment. At Fermilab we have modified the Jorway41 such that two interfaces may access (share) the same CAMAC branch. The new generation of experiments will read data from several sub-systems into a Microvax or $\mathrm{VAX}$ for logging to tape. The software reported in this paper allows them to read CAMAC data directly into the data siream on such systems.

\section{References}

1. JY4l1, CAMAC Branch Driver, Joruay Corporation.

2. DR11-W, Direct Memory Interface Module, Digital Equipment Corporation.

3. Charles Granjeri, Stanford Linear Accelerator, October 1978

4. User Interface and Internals of $V A X / V M S$ CAMAC Driver, Fermilab PN-217.

5. CAMAC/CDF-RABBIT Event Handier, Fermilab PN-218.

6. ANSI/IEEE Std. T58-1981, IEEE Standard Subroutines for CAMAC.

7. IEEE Routines for CAMAC and Extensións. Fermilab PN.219.

8. User Interface Routines for the CAMAC/CDF. RABBIT Event Handler, PN-220.

9. V. Whitc, et al. "High Specd Interprocessor Data Links using the DR1I-W presented at the 1982 Fall DECUS, C.S. Symposium. Anaheim. CA. 
10. FDL. A High Level Diagnostir Language for FASTBIS. IEEE Transactions on Nuclear science, Vol. NS-28. No. 5 , Oct. 1981; K-Nater Devire Diagnostic Language, D. Lesny, et al., Lniversity of Illinois, Fermilab, FBN022.

11. See companion paper to this conference. The VAXONLINE Software System at Fermilab, V. White, et al.

12. IEEE Standard CAMAC routines, D. Nitz, Univ. of Michigan.

13. See companion paper to this conference, Software for the Fermilab Crate Controller, M. Bernett, et al. 